\title{
Crack growth and microstructural changes in AISI 52100: white etching cracks (WEC) and fine granular area (FGA)
}

\author{
Stefan Averbeck ${ }^{1,1}$, Daniel Spriestersbach ${ }^{1}$, and Eberhard Kerscher $^{1}$ \\ ${ }^{1}$ Materials Testing, TU Kaiserslautern, Gottlieb-Daimler-Strasse, 67663 Kaiserslautern, Germany
}

\begin{abstract}
The service life of rolling element bearings can usually be predicted with good accuracy. However, the damage phenomenon ,white etching cracks" (WEC) can lead to premature and unforeseeable failures. The formation of zones with strongly decreased grain sizes at the crack faces is a characteristic of this type of failure. An earlier study showed that it is possible to reproduce this type of failure by multiaxial fatigue experiments with superposed cyclic compression and torsion. The present study shows the analysis of the stress state of those multiaxial fatigue tests with stress-based critical plane criteria. Using the critical plane results as a basis for a fracture mechanical analysis, a correlation is found between the crack length, the associated plastic zone size, and the extent of the transformed microstructure. This correlation is very similar to our results of the study of fine granular areas (FGA), a very high cycle fatigue phenomenon in bearing steels. It is argued that a plasticity-driven mechanism similar to the one proposed for FGA formation is responsible for WEC formation, too. Additional factors that are often cited as promoting WEC formation could explain the shift from very high cycle fatigue in the case of FGA to the early failures due to WEC.
\end{abstract}

\section{Introduction}

\subsection{State of knowledge on WEC and FGA}

The majority of rolling element bearings is manufactured from equivalents of the highcarbon alloy AISI 52100. The expected lifetime of such bearings can be calculated with the methods laid down in ISO 281, provided that proper operating conditions are maintained. Yet in some cases, premature bearing failures occur despite correct bearing design and operation. Metallographic examinations of these failed bearings reveal groups or networks of cracks near the contact surface which can cause spalling. After metallographic etching, it becomes apparent that the crack flanks are lined with zones of transformed microstructure which appear white under the optical microscope. This bearing damage phenomenon, called white etching cracks (WEC) or white structure flaking (WSF), has been reported from

$1 \quad$ Corresponding author: $\underline{\text { stefan.averbeck@mv.uni-k1.de }}$ 
many different applications (see reviews $[1,2]$ ) but is most prominent in wind turbine gearboxes [3]. It occurs regardless of bearing type, size, manufacturer, heat treatment, and other systematic causes.

Research activities of the past years have provided a detailed characterization of the transformed microstructure, which is found to be ferrite with extremely small grain diameters (10-100 nm). This and a supersaturation with carbon lead to very high hardness values of the white-etching zones. While it is difficult to determine the orientation of the nano-sized grains, the surrounding microstructure shows no preferred orientations. Further details can be found in [1, 2, 4-7].

The formation mechanism of WEC has been the subject of intense debate, and several hypotheses have been put forward. They offer different opinions on whether the cracks initiate at or below the surface, whether the cracks precede the microstructural change or vice versa, and to which degree the many possible influencing factors (e.g. slip, lubricant chemistry, electric effects ...) influence WEC formation [2]. The seemingly irreconcilable differences between some of the theories may, according to mounting evidence, arise because the term white etching cracks could refer to more than one damage phenomenon. If this is true, WEC in connection with axial surface cracks need to be distinguished from network-like subsurface WEC [2].

In a recent paper [8], a possible link between WEC formation and a fatigue phenomenon encountered in uniaxial very high cycle fatigue (VHCF) of high strength steels was discussed. That phenomenon, called fine granular area (FGA), bears striking resemblance to WEC in a number of ways. As the term FGA suggests, it consists of very fine grains compared to the original microstructure, with grain sizes of tens of nanometres and can cause failure at stresses below the traditional fatigue strength [9]. FGA can form during VHCF around defects like non-metallic inclusions and is coupled with crack initiation and propagation at stresses below the threshold stress intensity factor $K_{\text {th }}$. Similar to WEC, the formation mechanisms are not entirely clear, although many different hypotheses exist [10].

Recently, two of the authors developed further a plasticity-based mechanism [11] in which the crack growth inside the FGA and microstructural refinement are assumed to be interlinked $[12,13]$. This is based on the observation that crack growth in the FGA occurs at stress intensity factors (SIF) below the threshold SIF for long crack propagation, $K_{\text {th }}$, and that FGA formation only stops when the local SIF meets the long crack SIF threshold. Furthermore, the thickness of the fine-grained zones was compared to the estimated size of the plastic zone at the crack tip. Three plastic zone size models were used for this calculation (v. Mises, Tresca, Irwin). For static loads and plane strain conditions, the radius $r_{\mathrm{p}}$ of the plastic zone is calculated as follows:

$$
\begin{array}{lc}
\text { v. Mises } & r_{\mathrm{p}}=1 / 4 \pi^{-1}\left(K_{\max } / \sigma_{\mathrm{y}}\right)^{2}\left[3 / 2 \sin ^{2} \theta_{\mathrm{p}}+(1-2 v)^{2}\left(1+\cos \theta_{\mathrm{p}}\right)\right] \\
\text { Tresca } & r_{\mathrm{p}}=1 / 2 \pi^{-1}\left(K_{\max } / \sigma_{\mathrm{y}}\right)^{2} \sin ^{2} \theta_{\mathrm{p}} \\
\text { Irwin } & r_{\mathrm{p}}=1 / 6 \pi^{-1}\left(K_{\max } / \sigma_{\mathrm{y}}\right)^{2}
\end{array}
$$

$K_{\max }$ is the maximum SIF, $\sigma_{\mathrm{y}}$ is the yield strength of the material, $\theta_{\mathrm{p}}$ the angle to the crack plane, and $v$ is Poisson's ratio. For the present discussion, the plastic zone orthogonal to the $\operatorname{crack}\left(\theta_{\mathrm{p}}=\pi / 2\right)$ is relevant.

A cyclic or reversed plastic zone size can be calculated using the range of SIF $\Delta K$. This can lead to a much smaller estimation of the plastic zone size, only a quarter of equations (1)-(3). However, for a stress ratio of $\mathrm{R}=-1$, the two estimates are equivalent, as $\Delta K=2$ $K_{\max }$. This was the case in the experiments at the basis of [11] and therefore, the calculation of the cyclic plastic zone is not discussed in further detail. 
The results indicated a good match between the predicted plastic zone sizes according to equations (1) and (2) and the thickness of the FGA measured at crack surfaces and incipient VHCF cracks. The transformation of the microstructure was supposed to enable crack formation by lowering the local threshold for crack propagation, as $K_{\text {th }}$ is known to decrease with decreasing grain size.

\subsection{Background of the study}

Because of the similarities between WEC and FGA, this study aims to perform the analysis just described for cyclic compression-torsion experiments leading to WEC formation. Calculating SIFs poses some difficulties under multiaxial stresses, as a simple superposition of the different stresses often does not lead to correct results. In the present case, an additional obstacle is the compressional stress component. Many approaches to calculating multiaxial SIFs involve squaring the component SIFs, which would cause a compressional stress to have the same mathematical effect as a tensile stress. Experimentally, this is clearly not the case.

To circumvent this difficulty, a different approach was tested. The primary difficulty in calculating a SIF for the multiaxial stresses lies in properly characterising the stress $\sigma$. To analyse the stress conditions and crack behaviour, the multiaxial fatigue analysis approach of critical planes was used. An introduction to this type of analysis is given in $[14,15]$. A shared characteristic of many critical plane approaches is that they use a combination of the maximum normal stress $N_{\max }$ and shear stress amplitude $C_{\mathrm{a}}$ on the plane under consideration to determine a damage parameter in the form of an equivalent stress. The underlying principle can be expressed as:

$$
\sigma=C_{\mathrm{a}}+\kappa N_{\max }
$$

The factor $\kappa$ depends on the individual criterion and can be influenced by the stresses, in which case equation (4) becomes nonlinear. The orientation of the critical plane is described relative to an arbitrary coordinate system $x y z$. Two angles, usually designated $\theta$ and $\varphi$, describe the orientation of the normal vector of the critical plane.

Many critical plane criteria yield a stress value acting on the critical material plane(s). With this information, it is possible to calculate $K$ and/or $\Delta K$ for cracks in these planes. Such an approach does not seem to have been tried previously in the literature. The resulting SIF will be compared to the threshold SIF for crack propagation in the material and will be used to calculate the plastic zone size ahead of the crack tip. Finally, the paper aims to show that a similar mechanism as in FGA formation could be responsible for WEC formation, according to the results.

\section{Methods}

To study WEC formation, the cyclic stresses operating in a rolling element bearing were reproduced with in-phase cyclic compressional and torsional loads on a servohydraulic testing machine. The specimens used were made from AISI 52100 and heat-treated by bearing manufacturer SKF GmbH to match the material specifications of regular bearings. The geometry of the specimens was hourglass-shaped with a gauge length of $5 \mathrm{~mm}$ and gauge diameter of $3 \mathrm{~mm}$.

The compression load was applied with a stress ratio $R=-\infty$ while the torsional load was alternating $(R=-1)$. Such an approach was reported in [16] to simulate rolling contact fatigue conditions reasonably well. All tests were run in a chamber filled with a partially synthetic gearbox oil at $80{ }^{\circ} \mathrm{C}$. This oil is supposed to enhance WEC formation because of 
its additive package [17]. Each specimen was tested load-controlled at $10 \mathrm{~Hz}$ frequency up to $10^{6}$ load cycles, as WEC can be expected to occur by this time. After the experiments, the specimens were cleaned and examined using optical microscopy, scanning electron microscopy, and focused ion beam milling.

Out of the multitude of critical plane criteria that exist, the stress-based criteria by McDiarmid [18], Matake [19], and Susmel and Lazzarin [20] are used for the analysis. The choice is based on the reasoning outlined in [21]. Both the orientations of the predicted critical plane and the damage parameters were used to validate the criteria.

The plastic zone at the crack tip is calculated using the same models as were used in [11] (v. Mises, Tresca, Irwin). As the damage parameters of the critical plane approaches are calculated using the shear stress amplitude acting on a plane, it seems justifiable to assume that a range of stress intensity factor $\Delta K$ can be calculated using the damage parameter. Alternatively, the damage parameter could be used to calculate $K_{\max }$ on the plane.

\section{Results and discussion}

In this section, the experimental results and the critical plane analysis will first be shown and discussed in succession. As space is limited, only the most important findings can be presented. Additional information and results can be found in earlier publications by the authors, the most recent being [21]. The experimental and critical plane analysis results will be used for a more detailed fracture mechanics analysis.

\subsection{Experimental results}

While none of the specimens fractured during the experiments, the cyclic compressiontorsion load caused the formation of surface cracks on the specimens. An example is shown in Fig. 1. The cracks grew perpendicularly to the specimen axis up to a length of around 10 $\mu \mathrm{m}$. After reaching that length, the further crack propagation occurred at an angle of $0^{\circ}-45^{\circ}$ to the specimen axis. The average total length (tip to tip) of the cracks was around $50 \mu \mathrm{m}$.

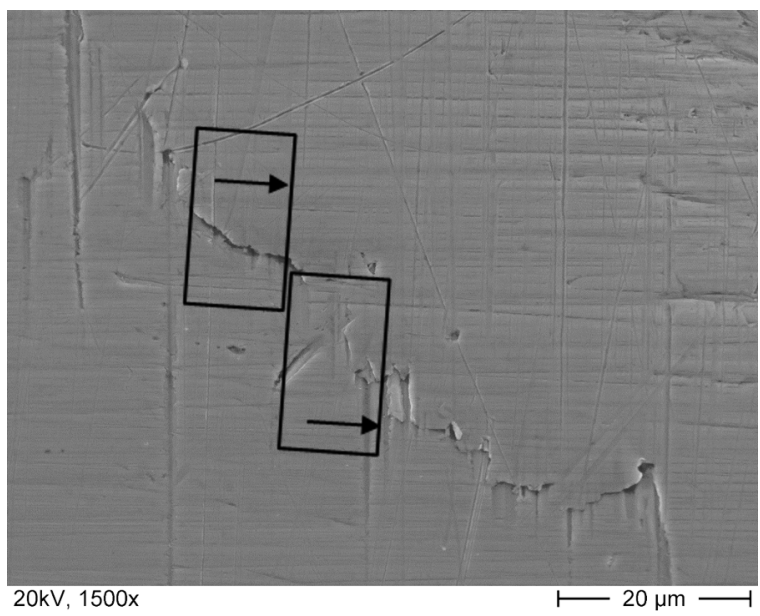

Fig. 1. Scanning electron microscope image of a surface crack with the positions of the FIB cuts shown in Fig. 2 (specimen axis from left to right).

The microstructure in the vicinity of the cracks was examined by focused ion beam (FIB) cross section cuts. Fig. 2 shows the images taken at the positions indicated in Fig. 1. The local microstructure at the crack tip seems to influence the crack propagation direction, 
which leads to a jagged crack. There is an overall tendency for the crack to propagate radially into the specimen at a very small angle $\left(<10^{\circ}\right)$. A layer of very small grains with diameters of a few dozen nanometres was found to accompany the cracks. The size of the grains increased in the direction of crack growth, and the larger grains near the crack tip appeared to be elongated parallel to the crack. However, these grains are still much smaller than the original grains, which are visible far away from the crack in the bottom of the images in Fig. 2. Due to the extremely small size of the grains, no precise quantitative data could be determined on the grain sizes.
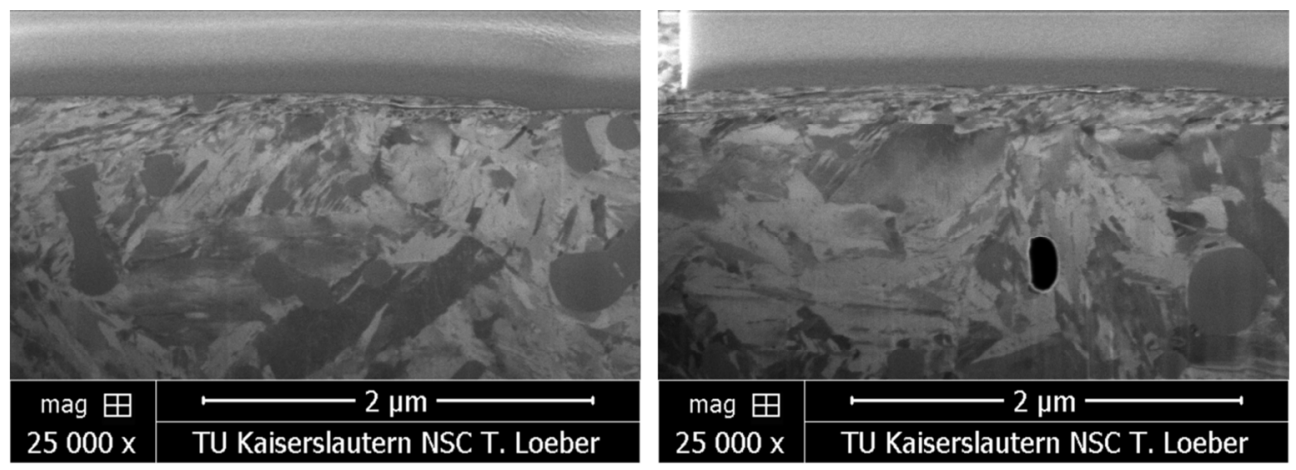

Fig. 2. Scanning electron microscope images of the fine-grained zones near the cracks.

From images like Fig. 2, the thickness of the fine-grained area can be measured at different positions along the crack path in order to analyse the development of the finegrained zones with increasing crack length. Due to limited FIB cut lengths, the cracks could only be traced up to a length of around $15 \mu \mathrm{m}$. As Fig. 3 shows, the thickness of the fine grained zone seems to increase with increasing crack length.

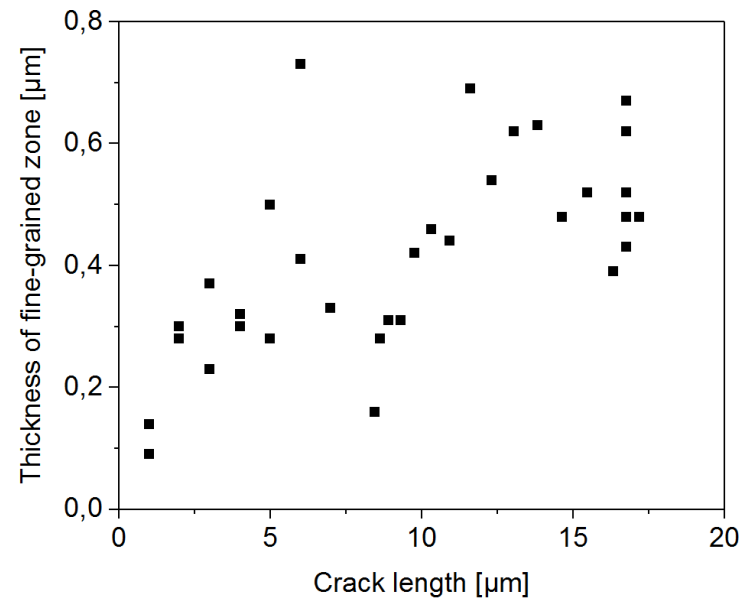

Fig. 3. Correlation between crack length and thickness of the fine-grained zone.

\subsection{Critical plane analysis results}

The three critical plane criteria used for this investigation predict similar critical plane orientations and damage parameters, as presented in Table 1. The angles $\theta$ and $\varphi$ specify the orientation of the normal vector of the critical plane relative to a coordinate system $x y z$, with $x$ parallel to the specimen axis. For a more illustrative interpretation, the orientations of the critical plane relative to the $x z$-plane, i.e. the plane tangential to the specimen surface, 
can be derived. They will be designated $\theta^{*}$ for the angle to the $x$-axis and $\varphi^{*}$ for the angle to the $z$-axis, respectively. For McDiarmid's criterion, $\theta^{*}=\varphi^{*}=7.32^{\circ}$, and for Matake's, $\theta^{*}=\varphi^{*}=3.95^{\circ}$. For Susmel and Lazzarin's criterion, the two angles are different from each other: $\theta^{*}=7.00^{\circ}$ and $\varphi^{*}=3.95^{\circ}$. For all criteria, the predicted orientations are very near the overall orientations of the cracks described in the previous subsection.

The damage parameters are well below the uniaxial fatigue limit of AISI 52100, which is around $900 \mathrm{MPa}$ at $10^{6}$ cycles when heat-treated to rolling element bearing specifications [22]. Thus, all criteria correctly predict that no failure occurs during the experiments.

Table 1. Results of the critical plane analysis.

\begin{tabular}{|c|c|c|c|}
\hline Criterion & First angle, $\boldsymbol{\theta}$ & Second angle, $\boldsymbol{\varphi}$ & Dmg. parameter \\
\hline McDiarmid & $90^{\circ}$ & $82.62^{\circ}$ & $486.61 \mathrm{MPa}$ \\
\hline Matake & $90^{\circ}$ & $86.04^{\circ}$ & $478.14 \mathrm{MPa}$ \\
\hline Susmel and Lazzarin & $90^{\circ}$ & $82.98^{\circ}$ & $488.20 \mathrm{MPa}$ \\
\hline
\end{tabular}

\subsection{Fracture mechanics analysis results}

With the damage parameters shown in Table 1 and the crack lengths measured from the SEM and FIB images, stress intensity factors can be calculated. For a crack length of $a=$ $10 \mu \mathrm{m}$, i.e. the distance which the crack grew perpendicularly to the specimen axis, the SIF $K_{\max }$ is between 1.74 and $1.78 \mathrm{MPa} \mathrm{m}^{1 / 2}$, depending on the criterion. This is well below the threshold SIF $K_{\text {th }}$ for long crack propagation in AISI 52100 of 4-5 $\mathrm{MPa} \mathrm{m}^{1 / 2}$. To meet this threshold, the crack length must exceed $21 \mu \mathrm{m}$. This means that the portion of the crack that is accompanied by fine-grained zones must have grown under sub-threshold SIF conditions.

In Fig. 4, the $K_{\max }$ values for the crack lengths shown in Fig. 3 are plotted against the corresponding thickness of the fine-grained zones, $t_{\text {WEC }}$. Additionally, the thickness of the FGA, $t_{\mathrm{FGA}}$. along VHCF cracks observed by [11] is shown with open symbols, while the plastic zone sizes from equations (3)-(5) are shown as lines. Note that the ordinate shows the total thickness of the transformed microstructure, meaning that the plastic zone size radius $r_{\mathrm{p}}$ from equations (1)-(3) must be multiplied by a factor of two to predict the diameter of the plastic zone.

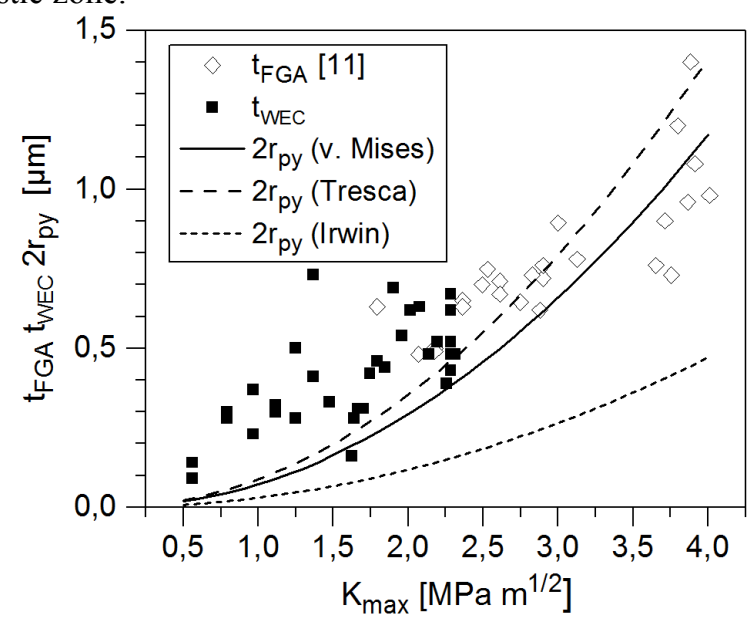

Fig. 4. Measured fine-grained zone thickness and calculated plastic zone size. 
The v. Mises and Tresca estimations for the plastic zone size are in good agreement with the measurements from WEC and FGA experiments. For WEC, a possible limitation arises from the use of the static plastic zone size. As the load ratio $R \neq-1$, the cyclic plastic zone in the experiments may be larger or smaller than in the static case. However, short cracks seem to behave very similarly no matter if the load is tensile or compressive and the closure stress intensity was very low in the compression experiments reported in [23].

Unlike the FGA experiments in [11], no lower limit of crack initiating SIFs was found in the WEC experiments. This could be explained by the different stress conditions or hydrogen from the lubricant, which is often cited to enhance plasticity in steels. A more detailed study of the maximum shear stresses on individual slip systems and their orientation under both loading conditions might deliver some insights.

The present results do not rule out the possibility of other formation mechanisms for both FGA and WEC. The hypothesis presented herein may only apply to a subset of damages in each category.

\section{Conclusions}

The fatigue phenomena fine granular areas (FGA) and white etching cracks (WEC) lead to the formation of cracks and microstructurally transformed zones in AISI 52100 bearing steel. While the loading conditions under which these phenomena evolve are quite different, they share a number of common properties. From this, the hypothesis arises that a similar formation mechanism may be responsible for both phenomena.

In the case of FGA, an earlier publication by two of the authors showed that a correlation is observed between the plastic zone size calculated from the local stress intensity factor and the thickness of the microstructurally transformed region. In the present study, critical plane approaches are used to calculate stress intensity factors and plastic zone sizes for WEC formed in cyclic compression-torsion experiments. As in the case of the FGA, the thickness of the microstructurally transformed region is closely predicted by the plastic zone size calculations according to v. Mises and Tresca. This supports the idea that WEC and FGA form by a similar mechanism: First, localised plasticity in the stress field at defects (later, at the crack tip) leads to grain refinement by dislocation rearrangement. This reduces the local threshold SIF, enabling crack propagation through the fine-grained area. As the crack grows, so does the plasticised volume, leading to an increasing thickness of the fine-grained zones. At a certain crack length, the global threshold SIF of the material is overcome, leading to long crack propagation without further grain refinement.

Some insecurity persists as to whether the damage parameter calculated from the critical plane criteria can readily be used to determine stress intensity factors, and whether the resulting SIF should be regarded rather as maximum SIF, $K_{\max }$, or range of SIF, $\Delta K$. Further study of this question is desirable, as is a more general inquiry into the usability of critical plane approaches for purely compressional loads.

The question why the very high cycle fatigue mechanism of FGA is moved to the high cycle fatigue region for WEC cannot be answered with certainty. It is supposed that influencing factors often cited as WEC-promoting (e.g. slip, lubricant additives/hydrogen, electricity) and the different stress state under rolling contact fatigue could play a role. This likely also causes the crack initiation SIF to drop below the limit of $1.7 \mathrm{MPa} \mathrm{m}^{0.5}$ reported in [11].

The authors would like to express their thanks to Dr. Thomas Löber of the Nano Structuring Center (NSC), TU Kaiserslautern, for the FIB preparation and imaging. This project was funded by the Deutsche Forschungsgemeinschaft (DFG) under grant KE1426/6-1. 


\section{References}

1. M.-H. Evans, White structure flaking (WSF) in wind turbine gearbox bearings: effects of 'butterflies' and white etching cracks (WECs), Tribol. Int. 28 3-22 (2012)

2. M.-H. Evans, An updated review: white etching cracks (WECs) and axial cracks in wind turbine gearbox bearings, Mater. Sci. Technol. 32 1133-69 (2016)

3. A. Greco, S. Sheng, J. Keller, A. Erdemir, Material wear and fatigue in wind turbine Systems, Wear 302 1583-91 (2012)

4. V. Šmel̦ova, A. Schwedt, L. Wang, W. Holweger, J. Mayer, Microstructural changes in White Etching Cracks (WECs) and their relationship with those in Dark Etching Region (DER) and White Etching Bands (WEBs) due to Rolling Contact Fatigue (RCF), Int. J. Fatigue 100 148-58 (2017)

5. Y.J. Li, M. Herbig, S. Goto, D. Raabe, Atomic scale characterization of white etching area and its adjacent matrix in a martensitic 100Cr6 bearing steel, Mater. Charact. 123 349-53 (2017)

6. H.K. Danielsen, F.G. Guzmán, K.V. Dahl, Y.J. Li, J. Wu, G. Jacobs, G. Burghardt, S. Fæster, H. Alimadadi, S. Goto, D. Raabe, R. Petrov, Multiscale characterization of White Etching Cracks (WEC) in a 100Cr6 bearing from a thrust bearing test rig, Wear 370-371 73-82 (2017)

7. O.H.E. West, A.M. Diederichs, H. Alimadadi, K.V. Dahl, M.A.J. Somers, Application of Complementary Techniques for Advanced Characterization of White Etching Cracks, Pract. Metallogr. 50 410-31 (2013)

8. F. Manieri, K. Stadler, G.E. Morales-Espejel, A. Kadiric, The origins of white etching cracks and their significance to rolling bearing failures, Int. J. Fatigue 120 107-33 (2019)

9. T. Sakai, Y. Sato, N. Oguma, Characteristic S-N properties of high-carbon-chromiumbearing steel under axial loading in long-life fatigue, Fatigue Fract. Eng. M. 25 765-73 (2002)

10. Y.-D. Li, L.-L. Zhang, Y.-H. Fei, X.-Y. Liu, M.-X. Li, On the formation mechanisms of fine granular area (FGA) on the fracture surface for high strength steels in the VHCF regime, Int. J. Fatigue 82 402-10 (2016)

11. D. Spriestersbach, E. Kerscher, The role of local plasticity during very high cycle fatigue crack initiation in high-strength steels, Int. J. Fatigue 111 93-100 (2018)

12. P. Grad, B. Reuscher, A. Brodyanski, M. Kopnarski, E. Kerscher, Mechanism of fatigue crack initiation and propagation in the very high cycle fatigue regime of highstrength steels, Scr. Mater. 67 838-41 (2012)

13. P. Grad, Rissinitiierung und Rissausbreitung im VHCF-Bereich des hochfesten Stahls 100Cr6 (TU Kaiserslautern, Kaiserslautern, 2013)

14. I.V. Papadopoulos, A comparative study of multiaxial high-cycle fatigue criteria for metals, Int. J. Fatigue 19 219-35 (1997)

15. I.V. Papadopoulos, Critical Plane Approaches in High-Cycle Fatigue: On the Definition of the Amplitude and Mean Value of the Shear Stress Acting on the Critical Plane, Fatigue Fract. Eng. M. 21 269-85 (1998) 
16. K. Burkart, Überrollungslebensdauer des Wälzlagerstahls 100 Cr6 in Abhängigkeit von nicht idealen Gefügeausbildungen unter besonderer Berücksichtigung der Karbidzeiligkeit (Universität Bremen, Bremen, 2009)

17. H. Surborg, Einfluss von Grundölen und Additiven auf die Bildung von WEC in Wälzlagern (Shaker, Aachen, 2014)

18. D.L. McDiarmid, A General Criterion for High Cycle Multiaxial Fatigue Failure, Fatigue Fract. Eng. M. 14 429-53 (1991)

19. T. Matake, An Explanation on Fatigue Limit under Combined Stress, Bulletin of JSME 20 257-63 (1977)

20. L. Susmel, P. Lazzarin, A bi-parametric Wohler curve for high cycle multiaxial fatigue assessment, Fatigue Fract. Eng. M. 25 63-78 (2002)

21. S. Averbeck, E. Kerscher, Critical plane analysis of multiaxial fatigue experiments leading to White Etching Crack formation, J. Phys.: Conf. Ser. 84312028 (2017)

22. A. Gabelli, J. Lai, T. Lund, K. Rydén, I. Strandell, G.E. Morales-Espejel, The fatigue limit of bearing steels - Part II: Characterization for life rating standards, Int. J. Fatigue 38 169-80 (2012)

23. R. Pippan, The growth of short cracks under cyclic compression, Fatigue Fract. Eng. M. 9 319-28 (1987) 